\title{
Fontes de fibra de coprodutos agroindustriais protéicos para coelhos em crescimento
}

\author{
Fiber sources of proteic agricultural co-products for growing rabbits
}

\author{
Marciana Retore ${ }^{\mathrm{I}}$ Leila Picolli da Silva ${ }^{\mathrm{II}}$ Geni Salete Pinto de Toledo ${ }^{\mathrm{II}}$ Ivan Graça Araújo ${ }^{\mathrm{II}}$ \\ Daniel Próis Eggers ${ }^{I I}$
}

\section{RESUMO}

Este trabalho teve por objetivo estudar a influência da fibra oriunda de diferentes fontes (feno de alfafa, farelo de linhaça e farelo proteinoso de milho) sobre o desempenho, coeficientes de digestibilidade e características da carne de coelhos em crescimento. Os animais foram distribuídos em um delineamento inteiramente casualizado com três tratamentos e oito repetições, sendo uma ração referência e duas rações testes: FA - ração controle, com feno de alfafa; FL - substituição total do feno de alfafa por farelo de linhaça e FP - substituição total do feno de alfafa por farelo proteinoso de milho. Os resultados foram submetidos à análise de variância e as médias comparadas pelo teste Duncan $(P<0,05)$. A maior quantidade de hemicelulose e a baixa capacidade de hidratação das dietas FA e FP proporcionaram maior peso vivo dos animais aos 89 dias (2123 e $1934 \mathrm{~g}$, respectivamente) contra $1797 \mathrm{~g}$ da dieta FL e melhor coeficiente de digestibilidade da MS e MO (média de 80,80 para $M S$ e $81,76 \%$ para $M O$, respectivamente) em relação à dieta $F L(73,86$ e $75,84 \%$, respectivamente). A dieta FP apresentou maior coeficiente de digestibilidade para a FDN (56,59\%) contra a média de $33,11 \%$ para as demais dietas. A força de cisalhamento foi menor para a carne dos animais alimentados com FA e FP (1,90 e 1,63 kgf) em comparação com a FL $(2,33 \mathrm{kgf})(P<0,05)$, o que indica carne mais macia. $O$ farelo proteinoso de milho proporcionou peso vivo dos animais aos 89 dias semelhante ao do feno de alfafa. O farelo de linhaça, pela grande quantidade de fibra solúvel e alta capacidade higroscópica, prejudicou o desempenho dos animais e a maciez da carne.

Palavras-chave: farelo de linhaça, farelo proteinoso de milho, feno de alfafa.

\section{ABSTRACT}

The objective of this paper was to study the influence of fiber fractions from different sources (alfalfa hay, linseed bran and corn gluten meal) on performance, digestibility coefficients and meat characteristics of growing rabbits. The animals were distributed into a completely randomized design with three treatments and eight replications, with one reference and two test diets: $\mathrm{AH}$ - control diet, with alfalfa hay; LB-total substitution of alfalfa hay by linseed bran and GM-total substitution of alfalfa hay by corn gluten meal. The data were submitted to variance analysis and the averages were compared by Duncan test $(P<.05)$. The largest amount of hemicellulose and the low hydration capacity of the diets AF and GM proportioned greater animals live weight at 89 days of age (2123 and $1934 \mathrm{~g}$, respectively) versus $1797 \mathrm{~g}$ of the animals fed with the diet $L B$ and better digestibility coeficients of DM and OM (average of 80.80 for DM and $81.76 \%$ for OM, respectively) in relation to diet $L B$ (73.86 and $75.84 \%$, respectively). The diet GM showed a higher digestibility coefficient of NDF (56.59\%) against the average of $33.11 \%$ for the other diets. The shear force was lower for meat from animals fed with $\mathrm{AH}$ and GM (1.90 and $1.63 \mathrm{kgf}$ ) compared to LB (2.33 kgf) $(P<.05)$, which indicates meat tenderness. The corn gluten meal proportioned live weight to the animals at 89 days of age similar to the alfalfa hay. The linseed bran, due to large amount of soluble fiber and high hydration capacity, impaired animal performance and meat tenderness.

Key words: alfalfa hay, corn gluten meal, linseed bran.

\section{INTRODUÇÃO}

O feno de alfafa é tradicionalmente incluído nas dietas de coelhos como fonte de fibra, além de contribuir com significativa quantidade de proteína, uma vez que contém, em média, 17\% de proteína bruta (HERRERA, 2003). A alimentação chega a representar

IPrograma de Pós-graduação em Zootecnia, Centro de Ciências Rurais (CCR), Universidade Federal de Santa Maria (UFSM). Av. Roraima, 1000, Bairro Camobi, 97105-900, Santa Maria, RS, Brasil. E-mail: maretore@hotmail.com. Autor para correspondência.

IIDepartamento de Zootecnia, UFSM, Santa Maria, RS, Brasil. 
$70 \%$ dos custos de produção, pois somente o feno de alfafa pode compreender cerca de $40 \%$ do custo da dieta (SCAPINELLO et al., 2003). Isso ocorre em função da falta de oferta deste alimento no mercado, devido às altas exigências de crescimento da leguminosa, especialmente com relação às condições de fertilidade do solo, boa drenagem e $\mathrm{pH}$ próximo à neutralidade, sendo estas características encontradas em poucas unidades de mapeamento de solos brasileiros (SILVA et al., 1995). Neste contexto, as pesquisas estão concentradas na busca por alimentos alternativos ao feno de alfafa na formulação de rações completas, os quais devem garantir otimização econômica e atendimento das exigências nutricionais dos animais.

Grande variedade de coprodutos oriundos do processamento de fontes vegetais (oleaginosas e cereais) está disponível no mercado sob a forma de tortas e farelos. Estes diferem pelo método de extração do óleo, onde a torta é obtida por meio de prensagem a frio, enquanto o farelo, além da prensagem, necessita de calor e solventes químicos. Porém, deve haver maior conhecimento sobre o valor nutritivo, objetivando seu melhor aproveitamento e utilização de forma mais eficiente, o que se refletirá na redução dos custos e na melhor produtividade (AZEVEDO, 1996).

Muitos ingredientes alternativos apresentam em sua composição, além de proteína e energia, elevados teores de fibra dietética com diferentes graus de solubilidade e composição química, o que pode ser fator determinante na sua utilização pelo animal, com destaque para o farelo de linhaça e o farelo proteinoso de milho na alimentação de coelhos. A linhaça é cultivada essencialmente para a produção de óleo que, após a sua remoção, dá origem ao farelo de linhaça. Apesar do seu considerável valor protéico, pesquisas com suínos e aves têm demonstrado que o uso do grão de linhaça ou de seus coprodutos afeta negativamente a utilização dos nutrientes da dieta, ganho de peso e conversão alimentar, especialmente quando os níveis de inclusão são aumentados (ORTIZ et al., 2001; SANTOS et al., 2005). Este fato pode ser atribuído à presença de mucilagem indigestível de alta hidratação (lignanas), aumentando expressivamente a viscosidade da digesta. De acordo com DE BLAS \& WISEMAN (1998), a capacidade de hidratação da fibra aumenta a viscosidade da digesta e diminui a digestibilidade dos nutrientes, dificultando a ação das enzimas e a difusão de substâncias ligadas ao processo digestivo. O farelo proteinoso de milho é a parte protéico-fibrosa do grão de milho que fica após a extração da maior parte do amido e do gérmen pelo processo empregado na produção do amido ou xarope, podendo conter também extrativos fermentados do milho e/ou farelo de gérmen de milho (LEITE, 2006). Segundo ALLEN \& GRANT (2000), este ingrediente pode conter de 40 a $45 \%$ de fibra em detergente neutro e apenas 3\% de lignina, além de ser uma fonte de fibra altamente digestível.

A maioria dos estudos objetivando avaliar ingredientes alternativos e seus efeitos sobre o desempenho de coelhos na fase de crescimento têm demonstrado grande variabilidade na eficiência alimentar, principalmente relacionado ao aproveitamento protéico, o que é influenciado pelo teor de frações diferenciadas de fibra e suas respectivas ações sobre a disponibilidade dos nutrientes (SANTOS et al., 2004). Diante do exposto, o presente trabalho teve por objetivo estudar a influência da fibra oriunda de diferentes fontes protéico-fibrosas (feno de alfafa, farelo de linhaça e farelo proteinoso de milho) sobre o desempenho, coeficientes de digestibilidade aparente e características da carne de coelhos submetidos às dietas experimentais.

\section{MATERIAL E MÉTODOS}

O ensaio foi conduzido no Laboratório de Cunicultura, do Departamento de Zootecnia, da Universidade Federal de Santa Maria, no período de 24 de setembro a 11 de novembro de 2007. Antes da formulação das dietas experimentais e início do ensaio biológico, os ingredientes testados (feno de alfafa, farelo de linhaça e farelo proteinoso de milho) foram analisados bromatologicamente, em duplicata, quanto à matéria seca, matéria mineral e lipídeos pelo método de BLIGH \& DYER (1959) e proteína bruta através da determinação de nitrogênio pelo método de Kjeldahl (N x 6,25), de acordo com as técnicas descritas pela AOAC (1995).

O teor de fibra total foi determinado conforme o método enzímico-gravimétrico nº 985.29 e $\mathrm{n}^{\circ} 991.42$ (AOAC, 1995). Os teores de fibra em detergente neutro (FDN), fibra em detergente ácido (FDA), celulose e lignina foram determinados de acordo com a metodologia de GOERING \& VAN SOEST (1970). O teor de hemicelulose foi calculado como a diferença entre o teor de FDN e FDA. O conteúdo de fibra solúvel foi determinado subtraindo-se a FDN da fibra total. As enzimas utilizadas para determinação da fibra total foram a $\alpha$-amilase, protease e amiloglicosidase. As propriedades físico-químicas de capacidade de ligação ao cobre e capacidade de hidratação foram determinadas pelo método de McBURNEY et al. (1983) e McCONNELL et al. (1974), respectivamente.

Com base na análise bromatológica dos alimentos (Tabela 1), formularam-se três dietas experimentais (Tabela 2), seguindo as recomendações nutricionais para coelhos das principais tabelas de exigências nutricionais (LEBAS, 1980; DE BLAS \& MATEOS, 1998), as quais estabelecem $15 \%$ para proteína bruta e 2500 kcal para energia digestível. No presente trabalho, os teores de proteína bruta e energia 
Tabela 1 - Composição bromatológica, fracionamento e propriedades físico-químicas da fibra dos coprodutos feno de alfafa, farelo de linhaça e farelo proteinoso de milho.

\begin{tabular}{lccc}
\hline & Feno de alfafa & Farelo de linhaça & Farelo proteinoso de milho \\
\hline Matéria seca (\%) & 87,72 & 89,34 & 84,77 \\
Cinzas (\%) & 8,32 & 6,45 & 6,92 \\
Proteína bruta (\%) & 19,10 & 33,05 & 27,59 \\
Lipídeos (\%) & 4,12 & 8,32 & 6,92 \\
Fibra total (\%) & 68,85 & 58,10 & 52,22 \\
FDN (\%) & 48,72 & 26,86 & 43,99 \\
FDA (\%) & 32,53 & 19,60 & 10,62 \\
Hemicelulose (\%) & 16,19 & 7,26 & 33,37 \\
Celulose (\%) & 27,85 & 14,70 & 13,96 \\
Lignina (\%) & 8,76 & 8,76 & 1,70 \\
Fibra solúvel (\%) & 20,13 & 31,24 & 8,23 \\
Capacidade de hidratação (g.g ${ }^{-1}$ ) & 5,80 & 7,5 & 2,67 \\
Capacidade de ligação ao cobre (mg.100g ${ }^{-1}$ ) & 555,70 & 496,90 & 488,40 \\
\hline
\end{tabular}

digestível foram fixados acima destes níveis, objetivando-se obter dietas com maior densidade nutricional, visto que as mesmas seriam fornecidas aos animais na forma farelada.
As dietas foram compostas majoritariamente por milho e farelo de soja, visando a substituição do feno de alfafa por coprodutos agroindustriais fontes de proteína: farelo de linhaça e farelo proteinoso de

Tabela 2 - Composição percentual e química das rações experimentais para coelhos em crescimento arraçoados na dieta com feno de alfafa, farelo de linhaça ou farelo proteinoso de milho.

\begin{tabular}{|c|c|c|c|}
\hline & Feno de alfafa & Farelo de linhaça & Farelo proteinoso de milho \\
\hline Ingredientes & \multicolumn{3}{|c|}{ 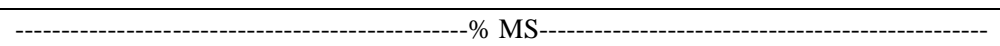 } \\
\hline Feno de alfafa & 15,00 & --- & --- \\
\hline Farelo de linhaça & --- & 20,00 & --- \\
\hline Farelo proteinoso de milho & --- & --- & 18,00 \\
\hline Milho & 52,46 & 50,00 & 48,47 \\
\hline Farelo de soja & 23,79 & 15,33 & 19,36 \\
\hline Casca de arroz & 5,00 & 8,50 & 7,40 \\
\hline Calcário calcítico & 0,38 & 0,90 & 0,80 \\
\hline Sal & 0,50 & 0,50 & 0,50 \\
\hline Suplemento Min e Vit* & 0,50 & 0,50 & 0,50 \\
\hline Fosfato bicálcico & 0,77 & 0,70 & 1,00 \\
\hline Óleo de soja & 1,60 & 3,57 & 4,00 \\
\hline \multicolumn{4}{|c|}{ m base na $\%$} \\
\hline $\mathrm{ED}(\mathrm{kcal} / \mathrm{kg})$ & 3.000 & 3.000 & 3.000 \\
\hline FDN & 22,00 & 22,50 & 23,30 \\
\hline FDA & 13,70 & 14,10 & 12,50 \\
\hline Hemicelulose & 8,21 & 6,86 & 11,38 \\
\hline Celulose & 9,85 & 9,33 & 8,71 \\
\hline Lignina & 3,92 & 4,75 & 3,15 \\
\hline Fibra solúvel & 5,21 & 6,74 & 6,96 \\
\hline Proteína bruta & 18,00 & 18,00 & 18,00 \\
\hline Cálcio & 0,70 & 0,73 & 0,76 \\
\hline Fósforo & 0,45 & 0,50 & 0,47 \\
\hline
\end{tabular}

* Composição por kg do produto: Vit A, 300.000 UI; Vit D, 50.000 UI; Vit E, 4.000 mg; Vit K3, 100 mg; Vit B1, 200 mg; Vit B2, 300 mg; Vit B6, 100 mg; Vit B12, 1.000 mcg; Ác. Nicotínico, 1.500 mg; Ác. Pantotênico, 1.000 mg; Colina, 35.000 mg; Ferro, 4.000 mg; Cobre, 600 mg; Cobalto, 100 mg; Manganês, 4.300 mg; Zinco, 6.000 mg; Iodo, 32 mg; Selênio, 8 mg; Metionina, 60.000 mg; Promotor de Crescimento, $1.500 \mathrm{mg}$; Coccidiostático, $12.500 \mathrm{mg}$; Antioxidante, $10.000 \mathrm{mg}$. 
milho. Essas rações formaram os tratamentos: FA - ração controle, com feno de alfafa; FL - substituição total do feno de alfafa por farelo de linhaça; FP - substituição total do feno de alfafa por farelo proteinoso de milho. Embora os tratamentos visassem a substituição do feno de alfafa, os demais ingredientes, como milho e farelo de soja, também tiveram suas proporções modificadas, a fim de se obterem dietas isoprotéicas e isocalóricas.

Foram utilizados 24 coelhos da raça Nova Zelândia Branco, independente de sexo, uma vez que o sexo dos coelhos não é fator influente no desempenho produtivo, desmamados aos 33 dias de idade com peso médio de $880 \mathrm{~g} \pm 82 \mathrm{~g}$, distribuídos aleatoriamente entre os tratamentos (8 animais/tratamento) e alojados em gaiolas individuais, com acesso livre à ração e água. $\mathrm{O}$ período de adaptação dos animais às dietas e instalações foi de sete dias. Após este período, os animais permaneceram submetidos às dietas experimentais dos 40 aos 89 dias de idade.

Ao longo dos 49 dias experimentais, ou seja, no primeiro dia experimental, aos 28 dias e aos 49 dias, foram feitas as pesagens das sobras de ração e dos animais para os cálculos de consumo de ração diário, ganho de peso diário e conversão alimentar. Para o ensaio de digestibilidade, utilizou-se óxido de cromo 0,05\%, misturado à ração, como marcador do início e término da coleta de fezes. A coleta total das fezes teve início 20 dias após o primeiro dia do experimento e foi realizada durante sete dias para estimar os coeficientes de digestibilidade aparente da matéria seca, matéria orgânica, proteína bruta e fibra em detergente neutro, de acordo com SCHNEIDER \& FLATT (1975).

No último dia experimental, após 12 horas de jejum, todos os animais, com 89 dias de idade, foram pesados, e metade deles foi abatida de acordo com a técnica de SCAPINELLO (1993). Para a obtenção da razão carne/osso, pesou-se e dissecou-se a perna traseira direita, segundo a metodologia descrita por RAO et al. (1978). Após a desossa, utilizou-se a carne para medir a perda de líquido por cozimento, conforme PILES et al. (2000). As amostras foram colocadas em bandejas de alumínio e assadas em forno elétrico a $250^{\circ} \mathrm{C}$ por 15 minutos, de modo a atingirem a temperatura interna em torno de $72^{\circ} \mathrm{C}$ (GAYA, 2006). A diferença em percentagem entre o peso final e o inicial correspondeu à perda de líquido por cozimento.

Para a determinação da maciez objetiva, as amostras destinadas à avaliação de perdas por cozimento, depois de assadas e resfriadas à temperatura ambiente, foram submetidas ao teste da força de cisalhamento. Procedeu-se a remoção de quatro amostras de 1,27cm de diâmetro de carne, as quais foram cisalhadas ao meio no equipamento "Warner-Bratzler
Shear" modelo 3000, conforme metodologia de KERTH et al. (1995).

O ensaio biológico foi conduzido em delineamento inteiramente casualizado, com três tratamentos e oito repetições, onde cada animal foi considerado uma unidade experimental. Os resultados obtidos foram submetidos a teste para detecção de valores anômalos (outlier) antes da análise de variância, sendo as médias comparadas pelo teste Duncan a 5\% de significância. A análise estatística foi realizada utilizando-se o Programa Estatístico SPSS versão 8.0 (Statistical Package for the Social Sciences) (1997).

\section{RESULTADOS E DISCUSSÃO}

Os coprodutos testados são de origem anatômofisiológica distinta e/ou de processamento (folhas, grãos e fermentados), assim, haverá grande diversidade estrutural de parede celular entre estas fontes vegetais, as quais se refletirão sobre as propriedades físicoquímicas da fibra. Portanto, independente do nível de inclusão de cada um desses coprodutos em relação ao milho e farelo de soja das dietas haverá alterações nos processos digestivos que repercutirão sobre o metabolismo animal.

Na fase dos 40 aos 68 dias de idade, o consumo de ração foi maior para os animais do tratamento FA(Tabela 3), o que refletiu no maior ganho de peso. O baixo consumo das outras dietas talvez possa ser atribuído à menor palatabilidade do farelo de linhaça e do farelo proteinoso de milho. No entanto, a conversão alimentar não diferiu entre os tratamentos FA e FP, provavelmente porque o farelo proteinoso de milho apresentou maiores teores de hemicelulose e pouca lignina, o que melhorou a digestibilidade dos nutrientes (Tabela 4). O tratamento FL proporcionou menor ganho de peso e pior conversão alimentar em relação às outras dietas, talvez em função da maior proporção de lignina em relação à celulose e hemicelulose, bem como, pelo fato da fração solúvel deste ingrediente possuir alta capacidade de hidratação (Tabela 1) e alta capacidade de formação de gel.

Considerando o período total do experimento, dos 40 aos 89 dias, não foi observado diferença para o consumo de ração. Todavia, o ganho de peso e conversão alimentar foi superior para os animais dos tratamentos FA e FP, possivelmente devido aos melhores coeficientes de digestibilidade dos nutrientes da dieta FP e, embora numericamente, do maior consumo de ração e melhor digestibilidade da dieta FA em relação ao tratamento FL, resultando no maior peso dos animais aos 89 dias.

Os animais do tratamento FL apresentaram o menor ganho de peso e pior conversão alimentar em 
Tabela 3 - Desempenho de coelhos aos 40, 68 e 89 dias de idade.

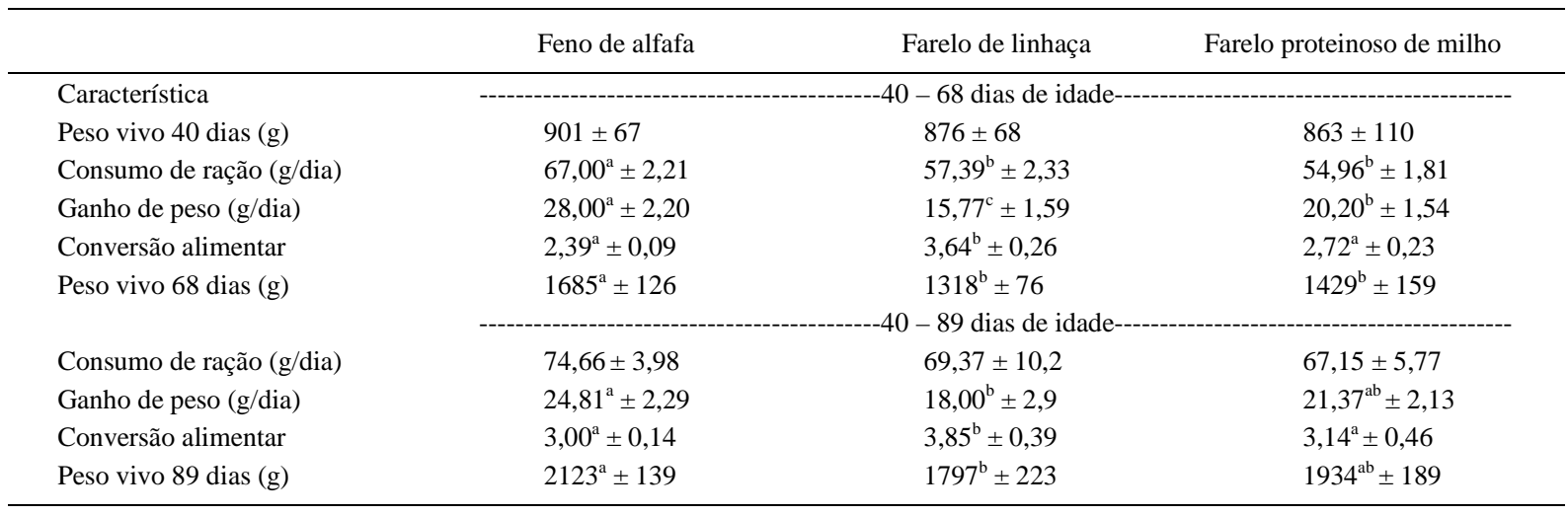

Resultados expressos como média \pm desvio padrão.

Médias seguidas de letras distintas, na linha, diferem significativamente pelo teste de Duncan $(\mathrm{P}<0,05)$.

relação aos demais, devido, em parte, à elevada capacidade de hidratação da fibra do farelo de linhaça (Tabela 1), o que afetou sobremaneira a digestibilidade dos nutrientes da dieta (Tabela 4).

De acordo com DE BLAS \& WISEMAN (1998), a capacidade de hidratação da fibra aumenta a viscosidade da digesta e diminui a digestibilidade dos nutrientes, dificultando a ação das enzimas e a difusão de substâncias ligadas ao processo digestivo. Esse fato é relatado em suínos e aves, onde as pesquisas mostram que o uso da linhaça ou seus coprodutos afeta negativamente a utilização dos nutrientes da dieta, ganho de peso e conversão alimentar, especialmente quando os níveis de inclusão desse ingrediente são aumentados (ORTIZ et al., 2001; SANTOS et al., 2005). Essa afirmação pode ser comprovada analisando-se a tabela 4, onde os coeficientes de digestibilidade aparente da dieta com inclusão de farelo de linhaça foram inferiores aos demais.

$\mathrm{Na}$ tabela 4 são apresentados os coeficientes de digestibilidade aparente das dietas experimentais. Segundo DE BLAS \& WISEMAN (1998), o efeito da fonte de fibra sobre a digestibilidade da matéria seca e matéria orgânica correlaciona-se diretamente com as características da parede celular do alimento fibroso. No entanto, os tratamentos FA e FP não diferiram quanto a estes coeficientes, embora o tratamento FP tenha apresentado numericamente melhores coeficientes de digestibilidade da matéria seca e matéria orgânica, visto que este ingrediente propiciou material menos lignificado, mais hemicelulose e, provavelmente, maior tempo de retenção cecal e atividade fermentativa.

Os coeficientes de digestibilidade aparente da proteína bruta não foram afetados pelo tipo de coproduto. Para os coeficientes de digestibilidade aparente da fibra em detergente neutro constatou-se melhor digestibilidade para a dieta contendo farelo proteinoso de milho (71\% superior aos demais tratamentos), pois esta dieta apresentou maior quantidade de hemicelulose e celulose em relação à lignina, quando comparado com os demais tratamentos.

As perdas por cozimento vão determinar o grau de maciez e suculência da carne. Quanto maiores as perdas, menor a maciez e mais seca a carne (OLIVEIRA \& LUI, 2006). Conforme apresentado na tabela 5, a razão carne/osso e as perdas de líquido por cozimento não diferiram entre os tratamentos. BOVERA

Tabela 4 - Coeficientes de digestibilidade aparente da matéria seca (CDMS), matéria orgânica (CDMO), proteína bruta (CDPB) e fibra em detergente neutro (CDFDN) das dietas experimentais para coelhos em crescimento.

\begin{tabular}{lccr}
\hline Parâmetros & Feno de alfafa & Farelo de linhaça & Farelo proteinoso de milho \\
\hline CDMS (\%) & $78,72^{\mathrm{ab}} \pm 4,85$ & $73,86^{\mathrm{b}} \pm 4,65$ & $82,87^{\mathrm{a}} \pm 2,54$ \\
CDMO (\%) & $79,90^{\mathrm{ab}} \pm 4,64$ & $75,84^{\mathrm{b}} \pm 4,34$ & $83,61^{\mathrm{a}} \pm 2,49$ \\
CDPB (\%) & $90,24 \pm 2,24$ & $89,60 \pm 2,76$ & $92,33 \pm 0,68$ \\
CDFDN (\%) & $32,65^{\mathrm{b}} \pm 2,28$ & $33,57^{\mathrm{b}} \pm 3,61$ & $56,59^{\mathrm{a}} \pm 4,04$ \\
\hline
\end{tabular}

Resultados expressos como média \pm desvio padrão.

Médias seguidas de letras distintas, na linha, diferem significativamente pelo teste de Duncan $(\mathrm{P}<0,05)$.

Ciência Rural, v.40, n.4, abr, 2010. 
Tabela 5 - Razão carne/osso (RC/O), perdas por cozimento (PCo) e força de cisalhamento (FC) da carne de coelhos abatidos aos 89 dias.

\begin{tabular}{lccc}
\hline Parâmetros & Feno de alfafa & Farelo de linhaça & Farelo proteinoso de milho \\
\hline RC/O (\%) & $4,33 \pm 0,43$ & $4,36 \pm 0,73$ & $4,58 \pm 0,36$ \\
PCo (\%) & $28,02 \pm 1,61$ & $30,60 \pm 3,86$ & $28,38 \pm 3,72$ \\
FC (kgf) & $1,90^{\mathrm{a}} \pm 0,17$ & $2,33^{\mathrm{b}} \pm 0,32$ & $1,63^{\mathrm{a}} \pm 0,15$ \\
\hline
\end{tabular}

Resultados expressos como média \pm desvio padrão.

Médias seguidas de letras distintas, na linha, diferem significativamente pelo teste de Duncan $(\mathrm{P}<0,05)$.

et al. (2008), ao avaliarem o consumo restrito e à vontade de coelhos em crescimento, obtiveram relação carne/ osso de 4,71 e $5,15 \%$, respectivamente, enquanto que a média do presente trabalho foi de $4,42 \%(\mathrm{P}>0,05)$. Os mesmos autores relataram perdas por cozimento de 29,15 e 27,73\%, respectivamente, valores semelhantes ao deste estudo. OLIVEIRA \& LUI (2006), também trabalhando com coelhos em crescimento, obtiveram relação carne/osso de 7,92 e perdas por cozimento de $26,89 \%$. A maior maciez verificada na carne dos animais que consumiram as dietas FA e FP é justificada pela maior velocidade de ganho de peso quando comparado aos animais da dieta FL.

\section{CONCLUSÃO}

O farelo proteinoso de milho proporcionou peso vivo dos animais aos 89 dias semelhante ao do feno de alfafa. O farelo de linhaça, pela grande quantidade de fibra solúvel e alta capacidade higroscópica, prejudicou o desempenho dos animais e a maciez da carne.

\section{REFERÊNCIAS}

AOAC. Official Methods of Analysis of the Association of Official Analytical Chemists. 16.ed. Washington, DC, 1995. 1018p.

ALLEN, D.M.; GRANT, R.J. Interactions between forage and wet corn gluten feed as sources of fiber in diets for lactating dairy cows. Journal of Dairy Science, v.83, p.322-331, 2000. Disponível em: <http://www.ncbi.nlm.nih.gov/pubmed/ 10714868>. Acesso em: 12 out. 2008. PMID: 10714868.

AZEVEDO, D.M.S. Fatores que afetam os valores de energia metabolizável da farinha de carne e ossos para aves. 1996. 68f. Dissertação (Mestrado em Zootecnia) Curso de Pós-graduação em Zootecnia, Universidade Federal de Viçosa, MG.

BLIGH, E.C.; DYER, W.J. A rapid method of total lipid extraction and purification. Canadian Journal of Biochemistry and Physiology, v.37, p.911-917, 1959. Disponível em: <http://dx.doi.org/10.1139/o59-099>. Acesso em: 12 jul. 2008 . doi: 10.1139/059-099.
BOVERA, F. et al. Feed restriction during summer: effect on rabbit carcass traits and meat quality. In: WORLD RABBIT CONGRESS, 9, 2008, Verona, Italy. Proceedings... Verona: WRSA, 2008. p.1325-1329.

DE BLAS, C.; MATEOS, G.G. Feed formulation. In: DE BLAS, C.; WISEMAN, J. The nutrition of the rabbit. London: Cab Publishing, 1998. p.241-253.

DE BLAS, C.; WISEMAN, J. The nutrition of the rabbit. London: Cab Publishing, 1998. 352p.

GAYA, L.G. Estudo genético da qualidade de carne em linhagem macho de frangos de corte. 2006. 127f. Tese (Doutorado em Zootecnia). Faculdade de Zootecnia e Engenharia de Alimentos - Universidade de São Paulo, SP.

GOERING, H.K.; VAN SOEST, P.J. Forage fiber analysis (apparatus, reagents, procedures and some applications). Washington, DC: USDA, 1970. 379p. (Agricultural Handbook).

HERRERA, A.P.N. Eficiência produtiva e avaliação nutricional de dietas simplificadas a base de forragens para coelhos em crescimento. 2003. 104f. Tese (Doutorado em Ciência Animal) - Universidade Federal de Minas Gerais, Belo Horizonte, MG.

KERTH, C.R. et al. Improvement of beef tenderness and quality with calcium chloride injection in beef loins 48 hours post mortem. Journal of Food Science, v.73, p.750-756, 1995. Disponível em: <http://www.ncbi.nlm.nih.gov/pubmed/7608007>. Acesso em: 30 mai. 2008. PMID: 7608007.

LEBAS, F. Les recherches sur l'alimentation du lapin: Evolution au cours dernieres années et perspectives d'avenir. In: WORLD RABBIT CONGRESS, 2., 1980, Paris. Proceedings... Paris: WRSA, 1980. V.2, p.1-17.

LEITE, D.T. Farelo de arroz desengordurado e farelo de glúten de milho na suplementação de bovinos de corte. 2006. 74f. Dissertação (Mestrado em Zootecnia) - Curso de Pós-graduação em Zootecnia, Universidade Federal de Santa Maria, RS.

McBURNEY, M.I. et al. Cation exchange capacity and buffering capacity of neutral-detergent fibres. Journal of the Science of Food and Agriculture, v.34, p.910-916, 1983. Disponível em: <http://dx.doi.org/10.1002/jsfa.2740340903>. Acesso em: 16 ago. 2008. doi: 10.1002/jsfa. 2740340903 .

McCONNELL, A.A. et al. Physical characteristics of vegetable foodstuffs that could influence bowel function. Journal of the 
Science of Food and Agriculture, v.25, p.1457-1464, 1974. Disponível em: <http://dx.doi.org/10.1002/jsfa.2740251205>. Acesso em: 15 jul. 2008. doi: 10.1002/jsfa.2740251205.

OLIVEIRA, M.C.; LUI, J.F. Desempenho, características de carcaça e viabilidade econômica de coelhos sexados abatidos em diferentes idades. Arquivo Brasileiro de Medicina Veterinária e Zootecnia, v.58, n.6, p.1149-1155, 2006. Disponível em: <http://dx.doi.org/10.1590/S0102-09352006000600025>. Acesso em: 16 jul. 2008. doi: 10.1590/S0102-09352006000600025.

ORTIZ, L.T. et al. Metabolisable energy value and digestibility of fat and fatty acids in linseed determined with growing broiler chickens. British Poultry Science, v.42, p.57-63, 2001. Disponível em: <http://www.informaworld.com/10.1080/ 713655010>. Acesso em: 20 ago. 2008. doi: 10.1080/ 713655010 .

PILES, M. et al. The effect of selection for growth rate on carcass composition and meat characteristics of rabbit. Meat Science, v.54, p.347-355, 2000. Disponível em: <http:// linkinghub.elsevier.com/retrieve/pii/S0309174099001096>. Acesso em: 10 ago. 2008. doi: 10.1016/S0309-1740(99)00109-6.

RAO, D.R. et al. Effect of weaning and slaughter ages on rabbit meat production. II. Carcass quality and composition. Journal of Animal Science, v.46, p.578-583, 1978. Disponível em: $<$ http://jas.fass.org/cgi/content/abstract/46/3/578>. Acesso em: 2 mai. 2008.

SANTOS, E.A. et al. Efeito dos níveis de fibra em detergente ácido sobre os coeficientes de digestibilidade das dietas e desempenho de coelhos em crescimento. Acta Scientiarum. Animal Sciences, v.26, n.1, p.79-86, 2004. Disponível em: <http://periodicos.uem.br/ojs/index.php/ActaSciAnimSci/ article/.../1932>. Acesso em: 20 jul. 2008. doi: 10.4025/ actascianimsci.v26i1.1932.

SANTOS, Z.A.S. et al. Valor nutricional de alimentos para suínos determinado na Universidade Federal de Lavras. Ciência e Agrotecnologia, v.29, n.1, p.232-237, 2005. Disponível em: <http://dx.doi.org/10.1590/S1413-70542005000100029>. Acesso em: 10 jul. 2008. doi: 10.1590/S1413-70542005000100029.

SCAPINELLO, C. Níveis de proteína bruta e de energia digestível e exigências de lisina e de metionina + cistina, para coelhos da raça Nova Zelândia Branco, em crescimento. 1993. 215f. Tese (Doutorado em Produção Animal) - Universidade Federal de Viçosa, MG.

SCAPINELLO, C. et al. Fenos de leucena (Leucaena leucocephala e Leucaena leucocephala cv. Cunningham) para coelhos em crescimento: digestibilidade e desempenho. Acta Scientiarum. Animal Sciences, v.25, n.2, p.301-306, 2003. Disponível em: <http://periodicos.uem.br/ojs/index.php/ ActaSciAnimSci/article/viewArticle/2006>. Acesso em: 16 ago. 2008. doi: 10.4025/actascianimsci.v25i2.2006.

SCHNEIDER, B.H.; FLATT, W.P. The evaluation of feeds throwgh digestibility experiments. Athens: University of Georgia, 1975. 423p.

SILVA, R.M. et al. Produção e qualidade da matéria seca de sete leguminosas estivais em Eldorado do Sul, RS. In: REUNIÃO ANUAL DA SOCIEDADE BRASILEIRA DE ZOOTECNIA, 32., 1995, Brasília. Anais... Brasília: SBZ, 1995. p.113-115.

SPSS para Windows. 1997. Versão 8.0.0. Chicago: SPSS [programa informático em CD-ROM]. Disponível em SPSS Inc. Página web de SPSS disponível em: <http://www.spss.com/>. 